\title{
Fundus Image Analysis to Detect Abnormalities in Diabetic Retinopathy using Computer Aided Design Tools - A Review
}

\author{
R. Lavanya, G. K. Rajini
}

\begin{abstract}
Diabetic retinopathy is becoming a major threat to visual loss in human beings. Many researchers are working to develop early detection techniques, which may reduce the risk of vision loss using image-processing techniques like image enhancement and segmentation. Improving the quality of medical images to detect the disease at an early stage is crucial for further medication. It is gaining more focus with automated techniques for machine learning. Filtering and morphological operators enhance image contrast and interested region can be extracted using segmentation techniques from the fundus image of the retina. For feature analysis the optical disk, localization of blood vessels and segmentation are very useful to observe the parameters like area, length and perimeter of blood vessels etc. Algorithms for this analysis include preprocessing, segmentation, feature extraction and classification. This paper tries to give a detailed review of various image-processing methods used in early detection of diabetic retinopathy and future insights to develop algorithms, which reduces clinician's time for diagnosis and pathogenesis.
\end{abstract}

Keywords: Diabetic retinopathy, Image enhancement, Pre-processing, IRE, Microanueurysm, Segmentation, Feature Extraction, Machine learning.

\section{INTRODUCTION}

D iabetic Retinopathy (DR) can be classified into two types namely Proliferative and non-proliferative. Abnormal vessel growth (also known as neovascularization) in retina refers to Proliferative Diabetic Retinopathy (PDR). The early stage of the disease without neovascularization is referred as Non-Proliferative Diabetic Retinopathy (NPDR). As the disease progress, it may develop into PDR causing severe vision problems, sometimes leading to permanent vision loss too [1]. A high level of blood sugar (known as Hyperglycemia) in humans causes diabetic mellitus like damage to capillaries of the retina. Early signs of diabetic retinopathy are Microanueurysm (small outpunching of retinal capillaries). Microanueurysm rupture and form as

Revised Manuscript Received on July 10, 2020.

* Correspondence Author

R.Lavanya1*, Department of ECE, NBKRIST/Research Scholar, VIT, Vellore, India. E-mail: lavanya.2017@vitstudent.ac.in

G. K. Rajini, Department of Instrumentation, VIT, School of Electrical Engineering, Vellore, India. E-mail: rajini.gk@vit.

(c) The Authors. Published by Blue Eyes Intelligence Engineering and Sciences Publication (BEIESP). This is an open access article under the CC BY-NC-ND license (http://creativecommons.org/licenses/by-nc-nd/4.0/) hemorrhages inside the retina weakening the vessels and cause fluid to seep into the retina. Fluid deposits under the macula termed as macular edema, which interferes with the normal vision slowly turns into PDR. NPDR is further divided into Early NPDR and Moderate NPDR. At least one Microanueurysm (small outpunching's) present in the retinal exam it gives signs of early NPDR. If more Microanueurysm present, then cotton wool spots known as Moderate NPDR characterize it and it leads to severe NPDR with venous bleeding and severe Intra-Retinal Micro Vascular Abnormalities (IRMA). Leaking of serum proteins and fatty deposits sometimes appear as cluster of microanueurysm found yellow in color in fundus retinal images and are known as exudates. Accumulation of lipids and proteins in the retina is termed as exudates. So abnormalities in the retinal images include detecting exudates and neovascularization are the useful features for automatic detection. As NPDR progresses, to bypass the damaged vessels, new vessel growth starts which is known as neovascularization. These new vessels are leaky and tenuous or brittle and sometimes misdirected and grow towards the vitreous causing sudden vision loss. Fundoscopy or Ophthalmoscopy allows clinicians to examine the retina, the optic disk and the underlying layer of blood vessels. As the disease progress, the diabetic retinopathy may cause vision loss [1], hence detection at an early stage is crucial. Computer-aided design tools play the major role to detect abnormalities at an early stage and help clinicians for proper diagnosis and treatment within less time [2, 3]. This paper tries to review various methods in literature to detect abnormalities in diabetic retinopathy at an early stage with the help of image processing techniques automatically. The following Fig.1. shows the disease progression from normal retina to proliferative retinopathy.

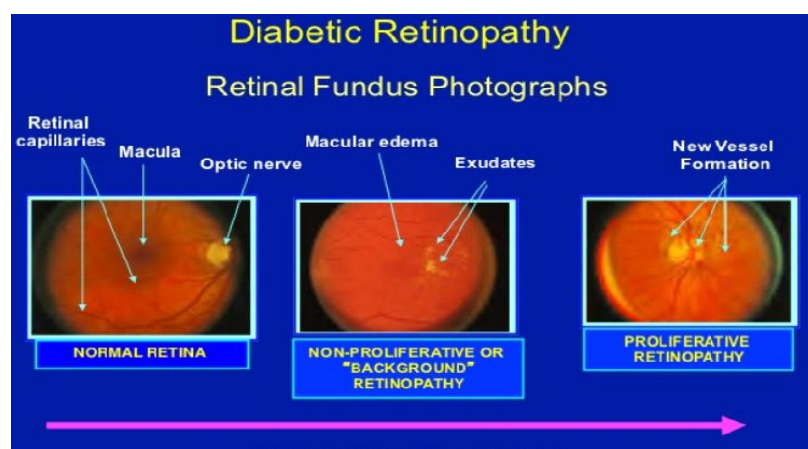

Fig. 1. Disease progression from the normal retina to the Diabetic retina

Blue Eyes Intelligence Engineering and Sciences Publication 


\section{METHODOLOGY}

Ophthalmoscopy or Fundoscopy is used to get the image of an eye in diabetic patients and these images are known as Fundus images. To get clear details of these, images are subjected to image processing techniques.

In general, various image-processing techniques like image denoising [14], enhancement and segmentation techniques are used to detect abnormalities. Generally, the acquired or captured images are applied to pre-processing techniques to enhance the contrast, brightness of the images, then Interested Region Extraction (IRE) i.e either Microanueurysm or exudates are obtained through segmentation and classification techniques, or the abnormalities can be detected using machine learning algorithms. Predictive modelings is to simplify pathology with the automatic computer-aided diagnosis like pre-processing, segmentation, classification and feature extraction with machine learning and are very much useful in the detection of the disease at an early stage. The general procedure includes pre-processing the acquired fundus image for image enhancement, segmentation and feature extraction for classification to detect abnormalities is given below Fig. 2 .

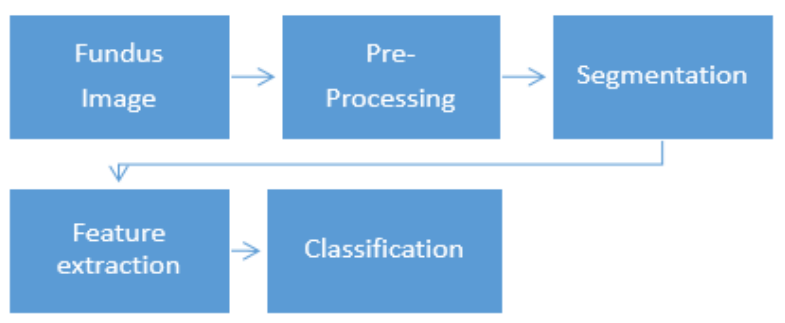

Fig. 2. General Flow of Retinal Image Analysis

\section{Pre-Processing:}

Retinal images from Fundoscopy are low contrast in nature and are not useful for detecting abnormalities by segmenting the selected or interested region. Generally available and most commonly used retinal databases are DRIVE (Digital Retinal Images for Vessel Extraction), STARE (Structured Analysis of Retina) DIARETDB (Diabetic Retinopathy Database), HRF (High-Resolution Fundus images database) and Messidor data sets. Fundus images are with poor contrast, non-uniform illumination, and noise. To increase the retinal image contrast, which is acquired from fundus camera many techniques are available in the literature, such as Local Histogram Equalization (LHE), Global Histogram Equalization (GHE) and Contrast Limited Adaptive Histogram Equalization (CLACHE). Pre-processing aims to highlight the interested region by enhancing the contrast. Various methods like spatial filtering techniques, which works on each pixel with different sizes of masks are available in literature. For enhancement, images from these databases are downscaled to overcome resolution and memory occupancy. Pre-processing steps include green channel extraction, downscaling and contrast enhancement making the image suitable for further processing. The accuracy of segmentation primarily relies on the consistency of the contrast of the entire image. Each pixel has its own importance for medical analysis, so without disturbing the image details contrast has to be increased. Many techniques have been proposed for enhancing low contrast images.
Histogram Equalization has its simplicity and good performance in almost all types of images. Histogram Equalization is classified into two classes: Global Histogram Equalization (GHE) and Local Histogram Equalization (LHE) techniques are used to detect lesions of blood vessels and the presence of optic nerve in the image. Image enhancement techniques magnify the interested region and noise, which causes overshoots. To eliminate overshoots in Laplacian Operator, inverse diffusion equation is used along with self-similarity filtering [6]. Fig. 3 shows enhanced images of diabetic retinopathy with Microanueurysm, blood vessels, and exudates.

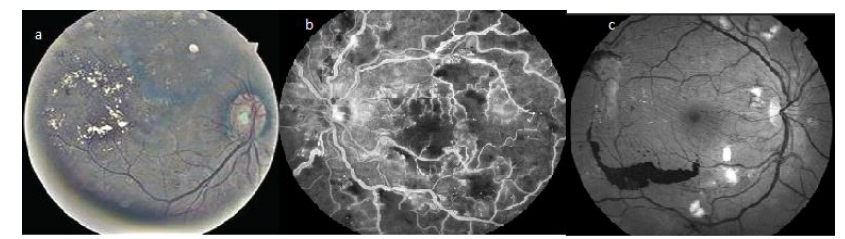

Fig. 3. Enhanced images of (a) Microanueurysm, (b) Blood vessels, \& (c) Exudates

Denoising the image before segmentation tries to enhance the features at interested region and the performance metric used in pre-processing is mainly to calculate Peak Signal to Noise Ratio (PSNR) and entropy. Time Domain Constraint Estimator (TDCE) is used calculate the PSNR of the retinal image without losing any details of the retinal image. Intensity information of fundus image is enhanced using Singular Value Decomposition (SVD) with help of sub bands of discrete wavelet transform [8], where it calculates entropy as the performance metric and compared with other methods like LHE, GHE, and SVE (Singular Values Equalization). DREAM tries to check the severity in the disease with pre-processing techniques to separate foreground from the retinal image, which consists of candidates or features to observe the severity of the disease [53] with help of spatial filtering to enhance the image. This paper mainly worked on reducing the selected features. In the pre-processing stage acquired images from fundus photography are resized to 256 X 256 or 512 X 512 and the green channel is extracted for more information and to make it clear for further processing.

Morphological operator Dilation is applied on the green channel and Contrast Limited Adaptive Histogram equalization (CLACHE) is used to enhance the image. In this paper, pre-processing includes green channel extraction, morphological processing, and contrast enhancement achieved through CLACHE [84]. To get the final pre-processed image, CLAHE image is subtracted from Gray image. Multi score second order Gaussian filters are used to enhance the vessels in pre-processing. As vessels are tiny structures, to get greater enhancement [37] uses Left Invariant rotating Derivative frame [LID], and Locally Adaptive Derivative frame [LAD] are used. The following table gives literature survey on preprocessing for enhancement.

\section{A. Segmentation}

Segmentation is a process of subdividing the image into multiple segments as the sets of pixels or super pixels to analyze in computer vision applications.

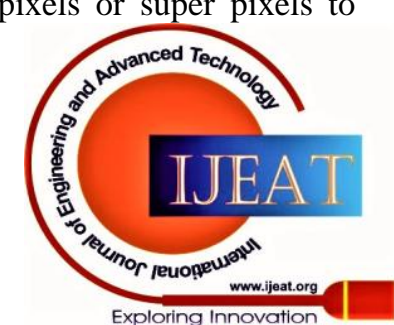


Segmentation is classified into three categories namely Supervised, Unsupervised and Semi-supervised. Supervised segmentation uses priori knowledge whereas unsupervised tries to find patterns of values and to build a probabilistic model

to identify

Table- I: Literature survey on pre-processing for image enhancement

\begin{tabular}{|c|c|c|c|}
\hline Image enhancement & Database & Method & Comments \\
\hline Satyardi[3],[6] & DRIVE, STARE & $\begin{array}{c}\text { Filter based technique's, } \\
\text { Gaussian Intensity distribution, }\end{array}$ & Improves contrast, loss of some image details \\
\hline Reza et al,[5] & DRIVE, STARE & Adaptive histogram & Improves local contrast as well as noise. \\
\hline $\begin{array}{l}\text { T.Jintasuttisak, } \\
\text { S.Intajag [10] }\end{array}$ & DRIVE, STARE & Histogram-based & $\begin{array}{l}\text { Improves contrast of the Green channel, } \\
\text { Non-uniform Illumination, amplifies noise too. }\end{array}$ \\
\hline Lavanya[13] & DRIVE & Cache filter & $\begin{array}{l}\text { Improves tiny structures, computational } \\
\text { complexity }\end{array}$ \\
\hline Xue et al,[7] & DRIVE, STARE, & $\begin{array}{c}\text { Gradient map \& } \\
\text { (LoG) Laplacian of Gaussian }\end{array}$ & $\begin{array}{l}\text { Local contrast features extracted with phase } \\
\text { Congruency }\end{array}$ \\
\hline Sarath Chandra[9] & $\begin{array}{l}\text { DRIVE, STARE, } \\
\text { Pvt. Database }\end{array}$ & $\begin{array}{l}\text { Histogram-based, Contour let } \\
\text { transform }\end{array}$ & Improved PSNR \\
\hline Miao et.al[11] & DRIVE, STARE & $\begin{array}{c}\text { Multiscale top hat } \\
\text { transformation, and linear } \\
\text { stretching }\end{array}$ & $\begin{array}{l}\text { Improved contrast in two levels, by extracting } \\
\text { bright features and optimized }\end{array}$ \\
\hline Xiao[12] & $\begin{array}{l}\text { DRIVE, Pvt } \\
\text { Database }\end{array}$ & Gamma correction & $\begin{array}{l}\text { Gray levels of the green channel are extracted, } \\
\text { the global contrast of the image is improved. }\end{array}$ \\
\hline
\end{tabular}

The substructures of the interested region in image analysis. Semi-supervised segmentation uses both labeled and unlabeled images of interested regions to identify abnormalities. In Diabetic Retinopathy, IRE may be Microanueurysm (MAs), Exudates (EXs) or Blood Vessels (BVs) and are application oriented.

Segmentation techniques are broadly classified based on an application, as if segmentation based on discontinuities (edge detection), or similarities (finding distance between the similar intensity pixels through connectivity based) using threshold based techniques. Segmentation methods are broadly categorized based on kernels, vessel tracking, thresholding, mathematical morphology and machine learning based [33, 41]. In segmentation process, Kirsch method applied for edge detection, which uses structuring element to find edges through neighbors. As each and every pixel is important to analyze medical images, for post-processing, dilation and erosion applied on segmented images to avoid the false detection of pixels. For retinal image analysis main features used to observe the abnormality are Exudates, Microanueurysm detection and blood vessel detection play crucial role in early detection of the disease.

(a) Microanueurysm (MAs): Microanueurysm is the first sign of visual impairments [16-26], detection of these is a trivial task which is close to blood vessels. Microanueurysm are focal dilatations of retinal capillaries with 10 to 100 micro meters. Microanueurysm detection is obtained by calculating size, height, and shape of the peak with a directional cross-sectional profile centered on the local maximum pixel.

Multiscale correlation with dynamic thresholding is used to detect MAs at coarse and fine levels to get candidate selection and true MAs [17]. SVD with contrast enhancement is applied on fundus images and the Hessian-based approach is used for candidate selection. Radon transforms with intensity normalization and SURF are used to locate the exact MAs candidate region [18]. Kernel-based techniques use the concept of vessel profile with intensity variations and also termed as matched filter techniques [13]. The performance metrics are specificity and sensitivity are achieved up to
$98.11 \%$ and $97.22 \%$ with the confidence level of 0.75 . Two types of segmentation methods are used to detect Microanueurysm effectively. They are coarse segmentation using morphological operators and fine segmentation with a Bayes classifier and achieved good results with 99. 9\% accuracy. Locally matching lesion templates are used to detect Microanueurysm from the sub-bands of wavelet-transformed images [19, 25]. The wavelet transform used for MAs detection, here templates are used to match lesions locally from the wavelet sub bands [57, 59]. 90.44\% sensitivity is achieved with Genetic algorithm as an optimization technique to select prominent features, which are used to observe abnormality in the given image. [20].

(c)Exudates (Exs): To get effective Exudate detection [28-35] Optical disc in the retinal image has to be eliminated using image segmentation. In paper [30] Exudate detection is achieved with an efficient integrated approach. The methodology includes various steps, first by enhancing the retinal image using Contrast Limited Adaptive Histogram Equalization, and then Hessian filtering separates blood vessels and the optical disk is identified with the combination of multiresolution analysis. In the remaining image fuzzy Kmeans, clustering is applied to detect exudates. K means clustering needs to specify the number of clusters and its effectiveness increases with maximum clusters, which in turn increases computational complexity. Exudates with the small radius are very hard to find, so Kirsch method tries to explore the edges in the retinal image. Linde-Buzo-Gray clustering is an improved version of $\mathrm{K}$ means algorithm by deriving good codebook for features, which detects the exudates by splitting the clusters but need improvements to find the location of cluster center [33]. This paper finds the bright lesions but fails to identify exudates with a small radius. Retinal image is pre-processed to improve accuracy in exudates detection, Exudate candidate regions are extracted with help of weighted ensembles, and these weights are determined using simulated annealing [34].

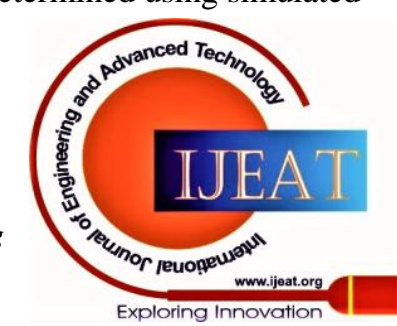


A novel approach proposed to get accurate borders of exudates, and exudates are identified using active contour method with the help of grey scale morphology and region wise classifier [35]. (b) Blood Vessels ((BVs): The structural details of blood vessels give maximum information to detect abnormalities in fundus images computer-aided tools. To detect vascular changes in blood vessels, vessels classified into arteries, veins and in turn graph based labels assigned to each vessel segment with the set of intensity features $[38,69]$.

By computing the geometric mean of the grey image of the diabetic retina, vessel orientation selectivity is achieved from the combination of difference Gaussian filters with unsupervised segmentation of blood vessels. For vessel detection, gradient is computed using different masks like Roberts, Prewitt, and Sobel operators. To describe global vascular structures of retinal blood vessels, intensity-based local features are employed to select the most prominent features with Expectation-Maximization optimization technique. For blood vessel segmentation Matched filter and fuzzy K , and median filters are deployed, and 96.43\% sensitivity 76.31\% specificity are achieved. Some researchers used Gaussian Intensity Distribution method for vessel segmentation. Blood vessel segmentation achieved with 94\% accuracy, $69 \%$ sensitivity and $97 \%$ specificity using morphological opening and binarization. Optical disc segmentation is achieved using domain knowledge with the low-rank representation of super pixels, and these methods employed for clustering. A new automated method developed to detect blood vessels, which uses directional differential operators to extract the skeleton of main vessel and vessel direction is emphasized using multi directional top-hat operator with rotating structuring element. The vessel information extracted with bit plane slicing and this information is integrated with main skeleton by applying morphological filters and iterative region growing method [86]. To detect the blood vessels, in pre-processing CLACHE algorithm and 2D Gabor filter with morphological reconstruction are used to achieve an average accuracy of $95 \%$. The multi-resolution analysis provides clear details of local binary patterns for texture analysis of blood vessels, which are also termed as uniform with computational simplicity [57]. Images are labeled on four-grade scale and features are extracted by isolating blood vessels, Microanueurysm and hard exudates using SVM to obtain 95\% sensitivity. To extract local details with more features from the non-diseased fundus image and these features must be invariant to illumination changes and orientation of pixels. These extracted features from normal image are matched with diseased fundus image to detect abnormalities. Scale Invariant Feature Transform (SIFT) is a local descriptor which uses grey scale images for local information based on scale pace [82]. The following Fig.4 represents segmentation of (MAs (a), EXs (b) and BVs(c).

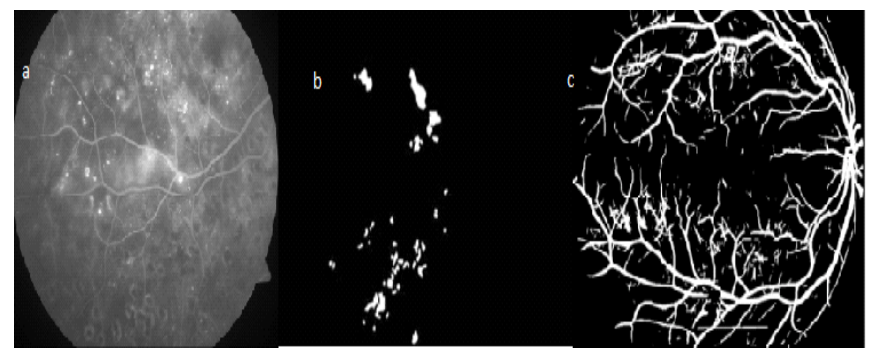

Fig. 4. Enhanced images of (a) Microanueurysm, (b) Blood vessels, \& (c) Exudates

\section{B. Feature extraction}

Feature is a general term given for an interested region depending on the application used to develop subsequent algorithm and repeatability, robustness, discriminating and independence are its main characteristics. Combination of features forms feature vectors, which were used to encode the image information. The feature vector includes the statistical parameters like entropy, homogeneity, and Euler number etc., with 64-point DCT [35]. Various features currently used in the literature are shape, color, textural and histogram of an image. As per the abstraction level, they categorized into pixel based, local or global based of an image. [60]. Feature extraction is a process of quantifying the actual information to the desired level and making it adaptable for further processing like classification and detection of abnormalities in the original image for analysis purpose. Feature extraction involves both identifications of relevant features and their selection. To get accuracy in findings, there is a great impact in the selection of local and global features from the image. Shape features are the area, perimeter, thinness ratio, aspect ratio, compactness ratio and elongation or eccentricity ratio. Color features give the contrast details of individual primary colors of Red, Green, and Blue. Histogram features give details of intensity levels of the entire image and their distribution. From the histogram, the following parameters like mean, standard deviation, skewness, energy, and entropy can be computed. Fourier descriptors give spectral details of an image and the parameter power is the primary metric for spectral features. Spectral features used to obtain textural details of the image and are of the function of image size related to the interested region. The texture of interested region has a significant role in medical image analysis and it can be categorized with the use of statistical methods depending on first and second order details like mean, variance, standard deviation, skewness and central moments etc., whereas the first order statistical details can be obtained from histograms. Second order details like correlation, homogeneity, energy and central moments obtained from Gray level co-occurrence matrix and Gray level Difference matrix. These are explained by Haralick to analyze the medical images [54]. Local Binary Pattern (LBP) is very much useful for spectral components and it is invariant texture analysis [59]. Model-based methods try to extract textural features through spatial properties to find the spatial relationships of textural features by using Markov models or Gibbs random models or Gaussian random models [65].

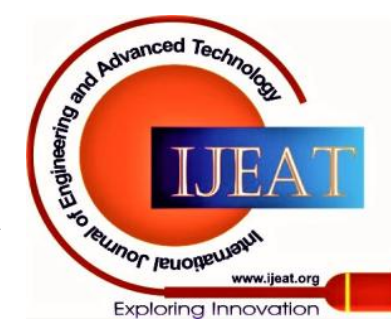


Texture analysis gives a reasonable amount of interested region to identify the abnormalities [53, 55, 57, and 58]. For vessel segmentation, the main categories are filter bank based methods, multiresolution analysis; Gabor filters [23] and Gabor wavelets. Among all Gabor and wavelets provides better information. Filter bank based methods works in frequency and spatial domains by using image transforms [38, 58].

Real-time applications need both frequency and spatial information, so wavelet methods are very much useful [20]. Multiple features may give robust results based on regional characteristics like regional variance, regional gradient, and regional spatial frequency, which explains using the Shearlet transform with the help of sub band coefficients [72, 79 \& 82]. While selecting features to classify an image, quality of features and interdependencies between features has gained importance [81, 83 \& 85].

Speeded Up Robust Features (SURF) are the framework of multiple scale analysis using convolution and local gradient [84] to extracts low level or local features like intensity and orientation [82]. This paper proposed a new ranking method SVM-RFE to observe quality with a reduced computational time with simple distance transform to estimate vessel width as small, medium and large for feature extraction and global intensity information are calculated using statistical measures.

\section{Classification}

Classification of retinal images to detect abnormalities is carried out using various classifiers. In supervised learning, the main classifiers are logistic regression and Naive bayes classifiers are linear models, which work with more independent variables. In medical images ground truth images are used to train the classifiers, whereas in unsupervised no ground truth images are available [78]. The main classification algorithms are the nearest neighbor, K-nearest neighbor, nearest centroid, template matching, Bayesian analysis and Neural based. Decision tree classifiers are non-parametric in nature and selection of a suitable classifier improves the classification accuracy. Knowledge-based classification plays a crucial role in medical imaging. The cost function is the measure observing the success rate of classification algorithms. In retinal image classification mostly used cost functions are Specificity (Sp), Sensitivity (Sn), Precision, Accuracy, and F-measure. Table.2 gives different types of supervised and unsupervised classifiers used for retinal image analysis.

Table- II: Types of Classifiers

\begin{tabular}{|c|c|c|}
\hline S.No & Supervised & Un-Supervised \\
\hline 1 & Linear Classifiers & Markov random Models \\
\hline 2 & Random Forest method & Pattern analysis \\
\hline 3 & Support Vector Machine & Fuzzy Clustering \\
\hline 4 & Decision Trees & Hierarchical Clustering \\
\hline 5 & Gaussian Mixture models & K-means Clustering \\
\hline
\end{tabular}

It is always good to have ground truth images properly labeled by the clinicians which are used to train the classifier to get good results, like supervised vector machines (SVM), K-means classifiers, GMM models and Ad boost , Nearest neighbors, neural networks, random forest, decision trees and SVM [65] are mostly used classifiers. Many researchers worked on SVM, which outperforms among all the classifiers.
Some researchers used neural networks for classification and Bayesian models too. Fuzzy C Means (FCM) clustering and SVM classifier is used to detect Diabetic Retinopathy and achieved Specificity of $95.83 \%$, accuracy of $96.7 \%$ with a sensitivity of $100 \%$. For binarizing input retina image line tracking concept is used and a hybrid technique with a combination of Scanning window analysis and Morphology is applied to get reliable results [33]. Neural networks are used for pixel classification and to detect Microanueurysm log Gabor features and Dual-Tree Complex Wavelet Transform are applied, then image feature is extracted by observing the difference between the intensity levels of the pixel with the pixel at the interested region [32]. This paper explains the local descriptors efficiently and termed as Local Contrast and Ordering (LCO) with higher computational efficiency. In neural networks, features of fundus image are applied as inputs to the number layers and network performance can be increased with a number of inputs, which increases computational cost, hence normalized inputs are used with iterative methods to reduce complexity in the network layers [62].

Multilayer Perceptron Neural Network [MLPNN], is used as a classifier which outperforms and achieves $100 \%$ training and cross-validation rates to detect abnormalities in the given image.. Pixel-wise classification is used to identify the maximum number of features from the vessels and the Genetic algorithm is used to optimize the features and achieved an accuracy of $90.2 \%$, sensitivity of $89.6 \%$ and specificity of $91.3 \%$ respectively [40].

Residual Networks (ResNets) are gaining popularity with minimum feature vectors and little complexity to get better segmentation results. Residual Fully Convolutional Network is proposed to predict segmentation by normalizing medical images with Fully Convolutional networks (FCN)[49], and they iteratively refined. Deep learning [75, 76 \& 77] methods are useful for supervised segmentation for retinal blood vessels and an accuracy of $99 \%$ and sensitivity of $87 \%$ are achieved. Hidden Markov models are an approach to trace the vessels accurately and occlusion handling [50]. The vessels are traced with an accuracy of $95.7 \%$ and specificity of $81 \%$. To diagnose PDR, matched filter and modified local entropy thresholding is applied to extract the blood vessels by achieving an average accuracy of $97.6 \%$ with Extreme Learning Machine [87]. Enhance K means algorithm is used for blood vessel segmentation and vessel features are extracted with the help of ROI based LBP method. The extracted features were classified by Echo State Neural Network \& RBF and achieved an accuracy of 97.95\% [88]. To observe global features of an image, histograms are very much useful and sometimes, Histogram distribution of different pixel class overlaps each other, which makes estimation of global parameters inaccurate. Estimation of local parameters with high accuracy is difficult due to inhomogeneity at different regions on spatial scale. To overcome this new method developed to calculate global parameters accurately [89].

Published By:

Blue Eyes Intelligence Engineering and Sciences Publication

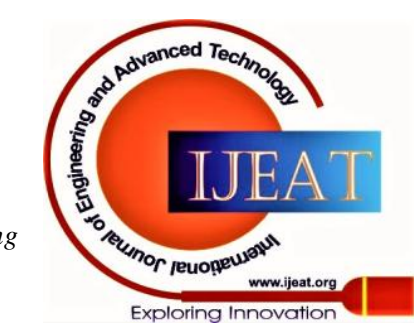


Fundus Image Analysis to Detect Abnormalities in Diabetic Retinopathy using Computer Aided Design Tools - A Review

One such method is Slope difference distribution for histogram is calculated and then global peaks identified. The local parameters calculated using Gibb's distribution, which characterizes image as markov model with joint distribution function and achieved good segmentation accuracy with an error rate of 5.7431 when compared to K-means 7.8177. The following Tables 3 and 4 summarizes literature review about various methods used for the analysis of abnormalities in Blood vessels (BV), Exudates (Ex) and Microanueurysm (MA).

Table III Various approaches in segmentation procedures

\begin{tabular}{|c|c|c|c|c|c|c|c|c|c|}
\hline \multirow{2}{*}{ Paper } & \multirow{2}{*}{ Author \& Year } & \multirow{2}{*}{\multicolumn{3}{|c|}{ Abnormalities }} & \multirow{2}{*}{ Database } & \multirow{2}{*}{ Method } & \multicolumn{3}{|c|}{ Performance Metrics } \\
\hline & & & & & & & Acc & Sp & Sn \\
\hline $\begin{array}{l}\text { Machine } \\
\text { learning } \\
\text { approach for } \\
\text { exudate }\end{array}$ & $\begin{array}{c}\text { Akara.Sophark } \\
2010\end{array}$ & MA & Ex & BV & N.A & $\begin{array}{l}\text { Naive } \\
\text { Bays+ } \\
\text { SVM }\end{array}$ & 98.14 & 92.28 & 98.52 \\
\hline $\begin{array}{l}\text { Automatic } \\
\text { Exudate } \\
\text { detection }\end{array}$ & $\begin{array}{c}\text { B.Harangi et.al } \\
2012\end{array}$ & - & - & Yes & Diaret DB1 & $\begin{array}{l}\text { Active } \\
\text { contour, } \\
\text { region wise } \\
\text { classifier }\end{array}$ & NA & NA & NA \\
\hline $\begin{array}{l}\text { Automatic } \\
\text { Exudate } \\
\text { detection }\end{array}$ & $\begin{array}{c}\text { Wuttichai } \\
\text { Laungruang,201 } \\
4\end{array}$ & Yes & Yes & Yes & DIARE TDB & $\begin{array}{c}\text { Hessian } \\
\text { filtering, } \\
\text { Fuzzy c } \\
\text { means }\end{array}$ & NA & NA & NA \\
\hline $\begin{array}{l}\text { Retinal vessel } \\
\text { segmentation } \\
\text { with ELM }\end{array}$ & C.Zhu.et al 2017 & Yes & Yes & Yes & DRIVE & $\begin{array}{c}\text { Phase } \\
\text { Congruency } \\
\text { \& ELM } \\
\text { classifier } \\
\end{array}$ & 96.07 & 98.68 & 71.4 \\
\hline DREAM & $\begin{array}{c}\text { Sohini } \\
\text { Choudhury } 2014\end{array}$ & Yes & Yes & Yes & $\begin{array}{l}\text { DRIVE, } \\
\text { STARE }\end{array}$ & - & 90 & 50 & 100 \\
\hline $\begin{array}{l}\text { Optimum } \\
\text { Wavelet } \\
\text { Transform }\end{array}$ & $\begin{array}{l}\text { G.W name et.al, } \\
2008\end{array}$ & Yes & Yes & Yes & $\begin{array}{c}\text { Pvt.Databas } \\
\text { e }\end{array}$ & $\begin{array}{l}\text { Wavelets } \\
\text { Genetic } \\
\text { algorithm }\end{array}$ & -- & -- & 89.92 \\
\hline
\end{tabular}

Table IV various approaches in segmentation procedures

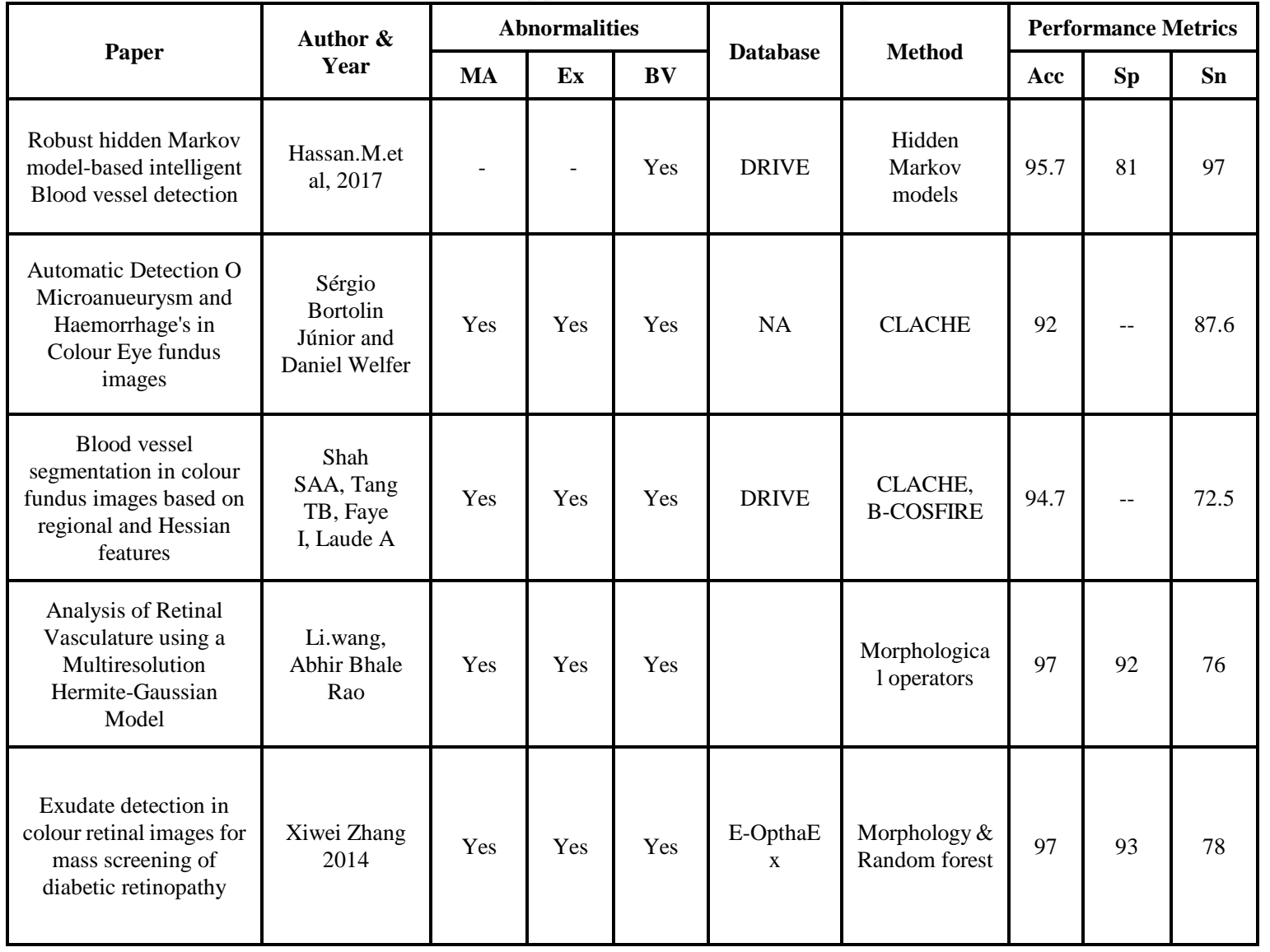

\section{CONCLUSIONS}

This paper explores various image processing techniques

Retrieval Number: C6366029320/2020@BEIESP DOI: 10.35940/ijeat.C6366.089620

Journal Website: www.ijeat.org
Published By:

Blue Eyes Intelligence Engineering and Sciences Publication 
and machine learning algorithms for detecting retinal abnormalities like the growth of abnormal vessels from normal vasculature, small Microanueurysm, and exudates, which may lead to permanent vision loss. Many researchers worked on pre-processing techniques to brighten the retinal structure for visual quality; still there is an ambiguity in standardizing the methodology to be followed.

As per the state of art, CLACHE technique outperforms in the pre-processing stage to improve contrast details, but a small amount of noise gets amplified and which is not recommended. In the segmentation process, the combination of connectivity based and thresholding techniques outperform with more complexity. In few papers, for classification of abnormality in the diabetic retinal images around 30 to 40 intensity, textural and statistical features observed for proper detection and there are no specific criteria for selecting a number of features to train the classifier. Some researchers used the maximum number of features with optimization techniques to obtain greater accuracy. Still, more work is needed to reduce the number of features to be used and to observe their effectiveness. The genetic algorithm used to optimize the number of features extracted and it is observed that instead of a single classifier, cascaded classifiers and semi-supervised learning outperforms for the given application. Now a day's deep learning, which is a subfield of machine learning, has been emerging as a prominent research area in retinal image analysis due to its effectiveness and less computational complexity. Still, improvements are needed to have accurate algorithms. Microanueurysm detection, Artery and vein classification of retinal images from fundus photography are giving more scope for future directions with proper selection of robust classifiers with suitable segmentation algorithm for computer-aided design. Hybrid methods like pixel-based segmentation with morphological operators are giving better results. This paper may give selection criteria for further research in retinal image analysis.

\section{ACKNOWLEDGMENT}

It is optional. The preferred spelling of the word "acknowledgment" in American English is without an "e" after the "g." Use the singular heading even if you have many acknowledgments. Avoid expressions such as "One of us (S.B.A.) would like to thank ... ." Instead, write "F. A. Author thanks" Sponsor and financial support acknowledgments are placed in the unnumbered footnote on the first page.

\section{REFERENCES}

1. Gordois A, Cutler H, Pezzullo L, Gordon K, Cruses A, Vineyard S, et al. "An estimation of the worldwide economic and health burden of visual impairment." Global Public Health. 2012;7(5):465-481.

2. Giancarlo L, Meriaudeau F, Karnowski TP, Li Y, Garg S, Tobin KW, et al. "Exudate-based diabetic macular edema detection in fundus images using publicly available datasets". Medical Image Analysis. 2012;16(1):216-226.

3. D. Satyarthi, M. R. Kumar, and S. Dandapat," Gaussian intensity distribution modeling of blood vessels in fundus images", Proceedings of the Annual IEEE India, 2005.

4. K.-Q. Huang, Q. Wang, and Z.-Y. Wu," Natural color image enhancement and evaluation algorithm based on the human visual system", Computer Vision, and Image Understanding, 2006( 103) pp. $52-63$.
5. Reza AM.' Realization of the contrast limited adaptive histogram equalization (CLAHE) for real-time image enhancement.' Journal of VLSI Signal Processing Systems for Signal, Image and Video Technology. 2004;38(1):35-44

6. Rampal H, Kumar RK, Ramanathan B, Das TP.” Complex shock filtering applied to retinal image enhancement." In: Proceedings of the World Congress on Medical Physics and Biomedical Engineering. Springer; 2013. p. 900-903.

7. Xue W, Mou X, Zhang L, Bovik AC, Feng X. "Blind image quality assessment using joint statistics of gradient magnitude and Laplacian feature"s. IEEE Transactions on Image Processing. 2014;23(11):4850-4862.

8. Lu Wang, Guohua Liu, Shujun Fu, LingZhong Xu, Kun Zhao, Caiming Zhang, "Retinal Image Enhancement Using Robust Inverse Diffusion Equation and Self-Similarity Filtering”.PLosOne, 2016

9. P.Sarathchandra, M.C Hanumanth Raju, M.T.Gopalakrishna,' Retinal Based Image Enhancement using Contourlet Transform”, Advances in Intelligent Systems and Computing, 2014, pp: 581-587

10. T.Jintasuttisak, S.Intajag," Color Retinal Image Enhancement by Rayleigh Contrast-Limited Adaptive Histogram Equalization”, in 14th International Conference on Control, Automation, and Systems, Korea, 2014, pp. 22-25.

11. Miao Liao, Yu-Qian Zhao, Xiao-hong Wang, Pei-shan Dai, Retinal vessel enhancement based on multi-scale top -hat transformation and histogram fitting stretching, Elsevier, 2014 pp. 56-62.

12. Xiao, Zhitao; Zhang, Xinpeng; Zhang, Fang; Geng, Lei; Wu, Jun; Su, Long; Chen, Liming, "Diabetic Retinopathy Retinal Image enhancement based on Gamma Correction", Journal of Medical imaging and health informatics, 2017(7), pp:149-154

13. R.Lavanya, P.Mohanaiah," A Hybrid Approach to visual vessel Extraction with Segmentation", Elsevier, Materials Today(8), 2018, pp:287-293.

14. Ahmad Fadzil.M.Hani et al.," Denoising Methods for Retinal Fundus Images", IEEE Xplore, 2014.

15. G.Tirupati*, B.Subba Rao, et al. "A Novel Technique for Fundus Image Contrast Enhancement”, International Journal of Bio-Medical Informatics and e-Health, Volume 2, No.1, 2014

16. Jonathan Goh, Lilian Tang et. Al," Evolving the Structure of Hidden Markov models for Microaneurysms detection", epubs.suvey.ac.uk, 2010

17. B.Zhang, X.Wu, J.You,\& F.Karry Detection of microanueurysm using Multiscale correlation coefficients, Pattern recognition. Vol.(43), pp:2237-2248,2010.

18. K.Adal, S.Ali, D.Sidibe, T.Kamaowski et al, "Automated detection of microanueurysm using robust blob descriptors". Medical Imaging, vol.8670, 2013.

19. Quellec.G, Mathieu land, et.al," Optimal Wavelet Transform for detection of microanueurysm in Retina Photographs", IEEE Transactions on Medical Imaging, vol.27, issue. 2008.

20. B.wu, W.Zhu, et al," Automatic detection of microanueurysm in retinal fundus images", Computer medical imaging \& Graphics, vol.55, pp.: 10-112, 2017.

21. Meindert.N.meijer, Bran van Ginneken ET. “Al Retinopathy online challenges: Automatic detection of microanueurysm in digital color fundus photographs", IEEE Transaction on Medical Image Analysis vol'29, issue1, pp.: 185-195, 2010.

22. T. Walter, P. Massenet et.al, "Automatic detection of microaneurysms in color fundus image, Medical Image Analysis, Vol. 11, pp. 555-566. 2017

23. Estevan lazar, Andras Hajdu Retinal microanueurysm detection through local rotating cross-section profile analysis", IEEE Transactions on Medical Imaging, vol.32, issue 2, 2013

24. Carla Peria, Diana Vega, "Using a multi-agent system approach for microaneurysm detection in fundus images”, Artificial Intelligence in medicine, vol.60: issue 3, .pp.17-188, 2014

25. Sujay, angadi \& Ravi Shankar, "Detection and classification of Microanueurysm using DTCWT and Log Gabor features in Retinal images”. Springer, Cham, Print ISBN: 978-3-319-12011-9.2010

26. Antal B, Hajdu A, An ensemble-based system for microaneurysm detection and diabetic retinopathy grading. IEEE Transaction on Biomedical Engineering. 59:1720-6, 2012

27. Leicht S.F. et.al., "Microaneurysm Turnover in Diabetic Retinopathy Assessed by Automated Retmarker DR Image Analysis - Potential Role as a Biomarker of Response to DR Treatment"', Ophthalmologica 231:198-203, 2014.

Published By:

Blue Eyes Intelligence Engineering and Sciences Publication

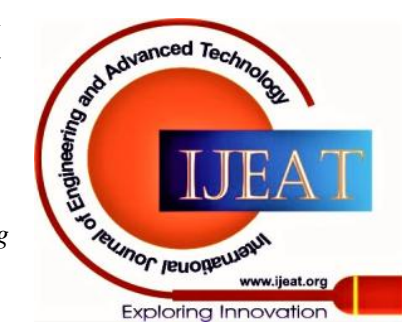


28. Akara Sopharak, "A Simple hybrid method for fine microaneurysm detection from non-dilated diabetic retinopathy retinal images", Computerized medical imaging and Graphics vol.137, issue 5-6, pp: 134-402, 2013

29. Oliver Faus et al, Algorithms for the Automated Detection of Diabetic Retinopathy Using Digital Fundus Images: A Review. Journal of Medical Systems, vol.36, issue 1, pp.: 147-157, 2012

30. Wuttichai Luangruangrong, Pusit Kulkasem, Suvanna Rasmequan, "Automatic Exudate detection in retinal images using efficient integrated approaches”. IEEE Xplore, 2015.

31. Akara sophark et al, Machine learning approach to Automatic exudate detection in retinal images from diabetic patients, Journal of Modern Optics, vol.57, issue 2, 2010.

32. B.Harangi, I.Lazar, A.Hajdes" Automatic exudate detection using active contour model \& region classification”, IEEE Explore, vol 12, 2012

33. Xiwei Zhang, Guillaume Thibault, Exudate detection in color retinal images for mass Screening of Diabetic Retinopathy, Medical image analysis Journal, vol.18, issue 7, pp.: 1026-1043, 2014.

34. P.Prentasic, S.Loncaric, "A Weighted ensemble based automatic detection of exudates in fundus photographs", Engineering in Medicine \& Biology Society (EMBC), 36th International Conference IEEE, 2014

35. Alan.D.Fleming Keith.A.Goatman "The mathematical morphology, the role of hemorrhage \& exudate detection in automatic grading Diabetic Retinopathy", British Journal of Ophthalmology, vol.94, issue 6, 2015

36. Feroui Atmel, Messadi Mohammad et al, Improvement of the hard exudate detection method used for computer-aided diagnosis, International Journal of image, Graphics \& Signal Processing, vol.4, and pp.: 1-27, 2012

37. N. Nimbarte et al. Comparative Analysis of Exudates Detection from Retinal Image with Effective Pre-processing Method, International Journal on Image \& Video Process. ISSN: 2336-0992, 2015

38. Zhang., Dashthozorg. "Robust retinal vessel segmentation via locally adaptive derivative frames in orientation scores", IEEE Transactions in Medical imaging vol.35( 12), 2016.pp. 2631-2644.

39. Argyrios Christodoulidis," A Multiscale tensor voting approach for small retinal vessel segmentation in high-resolution fundus image"s. PubMed 2016.

40. Gaze Pardo, et al, "Trainable COSFIRE filters for vessel delineation with application to retinal images", Medical Imaging Annals, vol.19, issue 1, pp.: 46-57, 2015.

41. Mohammad N. Nobi, Faruk Chowdhury et al, "A New Medical Image International Journal of Computer and Electrical Engineering, Vol. 3, No. 5, 2011.

42. Usman Akram, Ali Aziz et.al Blood vessel enhancement and segmentation using Wavelet Transform. Al, International Conference on Digital Image processing at Bangkok, Thailand, 2009.

43. Jyothiprava Dash \& Nilamani Bhoi, "A method for blood vessel segmentation in retinal images using morphological operators."International Conference on Computer, Electrical \& Communication Engineering, published in IEEE, 2016

44. S. Supot, C. Thanapong, P. Chuchart, and S. Manas, "Automatic segmentation of blood vessels in the retinal image based on fuzzy K-median clustering”, pp. 584-588, IEEE, Shenzhen, 2007.

45. D. Marín, A. Aquino et.al., "A new supervised method for blood vessel segmentation in retinal images by using gray-level and moment invariants-based features", IEEE Transactions on Medical Imaging, vol. 30, no. 1, pp. 146-158, 2011.

46. B.Dashbozorog, "An automatic graph-based approach for artery /vein classification in retinal images", IEEE Transactions on Image processing, vol; 23, issue 3, pp.: 1073-1083, 2014.

47. J.I.Orlando, M.B.Blaschko," Learning fully connected CRF's for blood vessel segmentation in retinal images", Medical image computing \& Computer-assisted intervention, lecture notes in computer science. Springer pp.634-641, 2014

48. L. Liskowski, K.Krawiec, "Segmenting retinal blood vessels with deep neural networks", IEEE Transactions in Medical Imaging vol.35 issue.11, pp.: 2369-2380. 2016

49. Cheng Zang, Zhu et al," Retinal vessel segmentation in color fundus images using extreme learning machine", Computerised Medical Imaging \& Graphics, vol.355, pp.: 68-77, 2017.

50. M.Hassan, Amin.M," Robust Hidden Markov models based on intelligent blood vessel detection of fundus images", Compute methods and programs in Biomedicine, vol.151, pp.: 13-201, 2017 Segmentation Technique Based on Variational Level Set Method",

51. M.D. Zeiler, Fergus, "Visualizing and Understanding Convolutional Networks", European Conference on Computer Vision, pp.: 818-833, Springer 2014

52. Ketki S. Arcade', Kshitija A et al., "Automatic Detection of Diabetic Retinopathy using Image Processing and Data Mining Techniques", International Conference on Green Computing and Internet of Things, IEEE, 2015

53. Sohini roychowdauri, "DREAM: Diabetic Retinopathy Analysis Using Machine Learning, IEEE Journal of Biomedical and Health Informatics, Vol. 18, NO. 5, 2014

54. Ananth madabhushi, Image analysis and machine learning in digital pathology: Challenges and Opportunitie"s, Ananth madabhushi, Medical image analysis, 2016

55. Ali Ahmadvand and Mohammad Reza Daliri, "A Review on Texture Analysis Methods" in Biomedical Image Processing, OMICS Journal of Radiology, OMICS J Radiol 5:2, 2016.

56. Haralick, R.M, Shanmugam K et al., "Textural features for image classification, Systems”, Man and Cybernetics, IEEE transactions pp: 610-621, 1973.

57. Ojala T, Pietikainen M, Maenpaa T, "Multiresolution Gray-scale, and rotation invariant texture classification with local binary patterns". Pattern Analysis and Machine Intelligence IEEE Transactions on 24: pp: 971-987, 2002

58. Ahmadvand A, Kabiri P," Multispectral MRI image segmentation using Markov random field mode"l. Signal, Image and Video Processing 10: pp: 251-258, 2016

59. Ahmadvand A, R, Hajiali MT, Mosavi MR,” A novel LBP method for invariant texture classification". In Second International Conference on Knowledge-Based Engineering and Innovation (KBEI) pp.: 152-157, 2015

60. Ahmadvand A, Daliri MR, "Rotation Invariant Texture Classification using Extended Wavelet Channel Combining and LL Channel Filter Bank". Knowledge-Based Systems, 2016

61. Bhateja V, Patel H et al," Multimodal Medical Image Sensor Fusion Framework Using Cascade of Wavelet and Contourlet Transform Domains”, Sensors Journal 15: pp:6783-6790, 2015.

62. Michael Drozdal et al", Learning normalized inputs for iterative estimation in medical image segmentation”, Medical Image Analysis, Vol.44, 2018

63. M.M El.Gayar, "A Comparative study of image low-level feature extraction algorithms". 2013

64. Lee.wang et.al ,'Analysis of Retinal Vasculature using a Multiresolution Hermite-Gaussian model", IEEE Transactions on Medical Imaging.

65. Harini.R \& Sheela.N, "Feature extraction and classification of retinal images for automated detection of Diabetic Retinopathy", Cognitive computing, and information processing, 2nd International IEEE conference 2016 .

66. Parth Panchal \& Tejendra Panchal "An algorithm for Retinal feature extraction using the Hybrid approach", Procedia, Computer Science Vol 79.2016.

67. Enrique.V.Carrera. et.al "Automated detection of diabetic retinopathy using SVM", Published in IEEE Xplore. 2017.

68. F.-L. Yi and W.-H. Xu, "Segmentation of blood vessels in color fundus images based on optimal multi-threshold method", Proceedings of the International Conference on Machine Learning and Cybernetics (ICMLC '12), vol. 2, pp. 725-728, IEEE, 2012

69. Malak.T.Buntan," Auto-segmentation of retinal blood vessels using Image processing”, DOI: 10.110/KACSTIT, Published in IEEE Xplore, 2016.

70. Xu Y, Duan L, Lin S, et.al,” Optic cup segmentation for glaucoma detection using low-rank super pixel representation", 2010

71. Jingyun duo et .al," LCO: A robust and efficient local descriptor for image matching", 2016

72. Xian Liu, Yue Zhou, Jiazun. "Image fusion based on shearlet transform and regional features", International Journal of Electronics \& Communication Vol.68.Issue 62013

73. Amole Prataprao Bhatkar, G.U.Karat, "Detection of Diabetic Retinopathy in Retinal Images Using MLP Classifier”, IEEE Xplore, 2016.

74. Xian Zhou, Jiajun Wang," Feature Selection for Image Classification Based on a New Ranking Criterion", Journal of Computer and C Zhang. Dashthozorg. Communications, vol.3, pp: 74-79, 2013

Published By:

Blue Eyes Intelligence Engineering and Sciences Publication 
75. Fan Huang, Bar.M.Ter. "Retinal artery/vein classification using genetic search feature selection", Computer methods \& programs in Biomedicine, vol.161, pp.: 197-207., 2018

76. K.K.Maini, J.put.Tuset, Deep retinal image understanding, Medical image computing \& Computer Assisted Intervention, Springer, and pp: 140-148.2016

77. I. Good fellow, Y.Benzio,” Deep Learning”, MIT press 2016.

78. Zhee, Zhou, et al., Zhee, Zhoy, et al., "Unsupervised segmentation of neutral images based on statistical modeling, Neuro Computing", vol.252, pp: 95-101, 2017

79. Ma, J., Sheridan, R. P., Liaw, A., Dahl, G. E. \& Svetnik,’Deep neural nets as a method for quantitative structure-activity relationships", V. J. Chem. Inf. Model. 55, 263-274 2015

80. MESSIDOR: Digital Retinal Images. http://messidor.crihan.fr/download-en.php.

81. Emanuale Trucco, Alfredo Ruggeri et.al. "Validating Retinal Fundus Image Analysis Algorithms: Issues \& purpose”, Investigating Ophthalmology and Visual Science, vol.54, pp: 3546-3559, 2013.

82. Jian $\mathrm{Wu}$, Zhiming cue et.al, A comparative study of SIFT and its invariants, Measurement Science Review, vol, 13. Issue.3, 2013.

83. Dawn.A.sim, Pearce.A.Kaene et.al "Automatic Retinal Image Analysis for Diabetic retinopathy in Telemedicine”, Springer, March 2015

84. Eduoard Oyallan, Julin Rabin, An analysis of SURF method, Image Processing Online, 5, pp. 176-218. 2015

85. Kirsty Tardon et.al," A review of feature-based retinal image analysis, Expert review of Ophthalmology", vol.12, issue3. 2017.

86. M.Fraz M .Basit A, Barman. S, "Application of morphological bit planes in retinal blood vessel extraction", Journal of Digital Imaging, 2013,26(2) PP: 274-286.

87. Bala.M.Ponni, Vijayachitra.S," Extraction of retinal blood vessels and diagnosis of Proliferative Diabetic Retinopathy using Extreme Learning Machine", Journal of medical Imaging and Health Informatics, 2015, vol 5, pp:248-256.

88. Nidhi.M.Tamil, Gunasekharan.K , "Efficient Ranking of Diabetic Retinopathy \& Glaucoma using Echo State Neural Network and Radial Basis function,'Journal of medical Imaging and Health Informatics, 2016, vol 6, pp: 869-874.

89. Wang, Zhen Zhou ," Image segmentation by combining global \& local properties” Expert Systems with Applications, 2017, vol 87, pp:30-40.

\section{AUTHORS PROFILE}

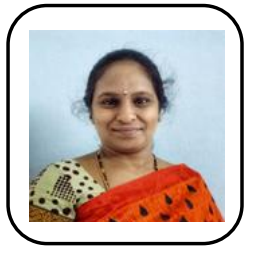

Smt. R.Lavanya, research Scholar at VIT, Vellore Currently working as Asst.Professor in the Dept of Electronics \& Communication Engineering, NBKR Institute of Science and Technology, Vidyanagar since 2006.. She completed her graduation in Electronics and communication at Narayana Engg College, under JNTU, Hyderabad, and her Post graduation in Digital Systems and Computer Electronics at JNTUA, Ananthapur. She conducted many national technical symposiums at student level. Published more than 7 papers in national and international journals. Her recent works are Retinal vessel Feature extraction from fundus image using image processing Techniques in Journal of Engineering and Technology(UAE), 2018, and A hybrid approach to visual vessel extraction with segmentation in Science Direct (2018).Her research interests include Bio medical imaging, wireless communications and Internet of Things and Machine learning.

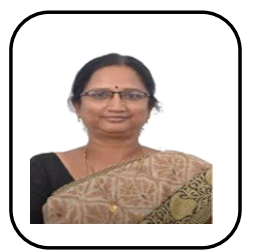

Dr. Rajini G.K, currently working as Associate Professor at VIT, Vellore. She completed her $\mathrm{PhD}$ at SV University Tirupathi on Image and Signal processing. Having 23 years of experience in teaching, she published more than 20 papers in national and international Journals. She worked in reputed engineering colleges located at Andhra Pradesh \&Tamil Nadu. . Her research interests include Signal \& Image processing, Bio medical imaging and wireless communications. Her recent works on Diagnosis of abnormal heartbeat using wavelet transform published by IEEE and High efficient dense field and all in-focus compression for loss less satellite image by using CCSDS in International journal of speech technology. She guided many Unger graduate and Post Graduate Projects. She organised many workshops and attended more than 20 national \& international Seminars and conferences. She is a good counsellor and motivator for her student community.

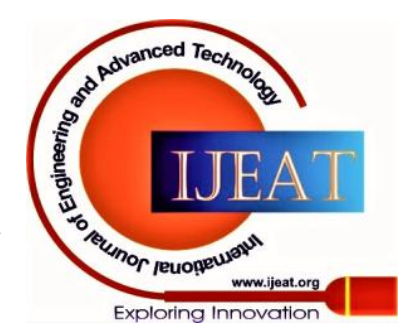

